
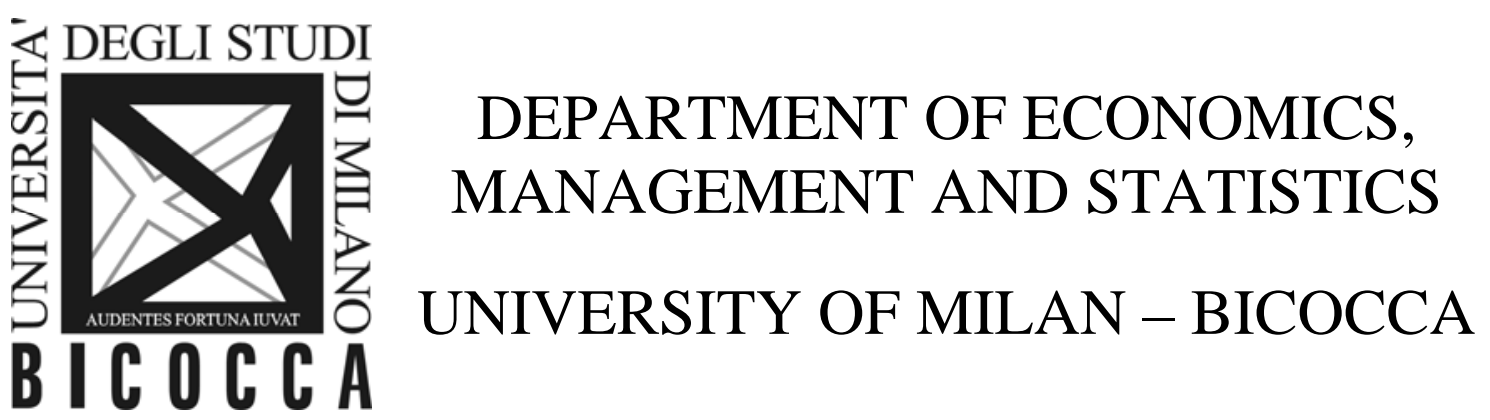

DEMS WORKING PAPER SERIES

\title{
The Macroeconomic Determinants of the US Term-Structure during the Great Moderation
}

Alessia Paccagnini

No. 274 - June 2014

Dipartimento di Economia, Metodi Quantitativi e Strategie di Impresa Università degli Studi di Milano - Bicocca 


\title{
The Macroeconomic Determinants of the US Term-Structure during the Great Moderation ${ }^{1}$
}

\author{
Alessia Paccagnini $^{2}$
}

May 30, 2014

\footnotetext{
${ }^{1}$ I am grateful to Imène Kouki, Antti Ripatti, and all participants of the 3rd International Symposium in Computational Economics and Finance (ISCEF).

${ }^{2}$ Università degli Studi di Milano - Bicocca, email: alessia.paccagnini@unimib.it
} 


\begin{abstract}
The aim of this paper is to study how the macroeconomic impulses can affect the term structure during the Great Moderation. As novelty in the research strategy, we create a term-structure using three latent factors of the yield curve. A Nelson-Siegel Model is implemented to estimate the latent factors which correspond to the level, the slope, and the curvature of the yield curve. As policy implication, the interpolated term structure suggests us how all the macro shocks impact on the overall yield curve, even if the impact has a different magnitude across maturities.

JEL CODES: G12, E43, E44, E58

KEYWORDS: Term structure of interest rates, yield curve, VAR, Factor Model
\end{abstract}




\section{Introduction}

The term structure of interest rates and macroeconomic variables are closely related: the real activity and expectations of future inflation can be important determinants of the yield curve. Several studies evidence the link between macro and term structure using latent factors (Ang and Piazzesi (2003), Diebold and Li (2006), Diebold et al. (2006), Mumtaz and Surico (2009), Bianchi et al. (2009), Gasha et al. (2010), and Medeiros and Rodriguez (2011)). Hence, using a term structure with different maturities, it is possible to extract latent factors, such as the level, the slope, and the curvature, to summarize the main features of the yield curve.

In this paper, we ask how the macroeconomic impulses can affect the US term structure during the Great Moderation period, before the Great Financial Crisis of 2007-2009 ${ }^{1}$. The Great Moderation period, spans from 1984 to 2007, was a calm down era in the US economy both for macroeconomic and for financial variables, even if in the same period there were several international financial crises (such as, the financial crisis in the South-East Asia countries in 1997 and in the Russia in 1998, and the Argentine economic crisis in late 90s, see Reinhart and Rogoff, 2009 for more details). The choice of this historical period is lead by a simplicity reason. We focus our empirical analysis on the methodology of the latent factors to investigate how the macroeconomic variables can affect the overall term structure interpolated using the factors. Consequently, for simplicity, we aim the empirical assessment only on US economy avoiding to consider spillover and global interactions with other economies. Moreover, considering only the Great Moderation period, we can ignore the changes in regime and time-variation which need to be studied using specific econometric tools as shown in Mumtaz and Surico (2009) and Bianchi et al. (2009).

\footnotetext{
${ }^{1}$ Several papers discuss the impact of the Financial Crisis of 2007-2009 on the term-structure and on the spreads (see, Medeiros and Rodríguez, 2011; De Pace and Weber, 2013; Cenesizoglu, Larocque, and Normandin, 2013; and Contessi, De Pace, and Guidolin, 2014)
} 
We aim to investigate how the maturities of a term structure respond to the key macro variables shocks in an Impulse-Response Functions (IRFs) exercise, highlighting a common pattern. As first step, we implement a preliminary analysis with a VAR set-up to report the responses to macro shocks of a term structure of seven maturities. The IRFs analysis suggests us a clear impossibility to define a common behavioral from the overall term structure to a specific macro shock. Since, the term structure depicts a set of yields on US Treasury securities of different maturities, focusing on the relationship among short-, medium-, and long-term yields; hence, we need a term structure with several maturities. The sample of the observed yields is not enough complete in terms of maturities, for example the yield series for 1-month Treasury bond starts only from 2001. Hence, our preliminary analysis suffers from this problem. To recover a more complete US Treasury yield curve, we use a latent factor no-arbitrage model which, in addition, exploits the relationship between these factors and the macroeconomic variables that underlie the term structure.

The finance literature tends to focus on two models of the term structure, namely the Nelson-Siegel Models, or NSMs, and Affine-Term Structure models, or ATSMs (Diebold, Piazzesi, and Rudebusch, 2005; Van Deventer, Imai, and Mesler, 2005; Baz and Chacko, 2004; and Bolder, 2001). These models of the term structure attempt to replicate an observed yield curve. On one side, in the NSMs, we rely on latent factors which are the parameters of a class of mathematical approximating functions. These models can also include observable macroeconomic variables, reflecting the importance of the joint behavior of the yield curve and macroeconomic variables for bond pricing, investment decisions, and public policy (Ang and Piazzesi, 2003). On the other side, the ATSMs include some of the traditional term structure models in the finance literature, including the general singlefactor model, the Cox- Ingersoll-Ross (CIR) model, and the multi-factor model.

We concentrate the empirical analysis on the NSMs. Diebold and Li (2006) discuss the 
powerful of these models which can account for the existence of unobservable, or latent factors and their corresponded factor loadings and key economic variables. The three factors are compared to their empirical counterparts, i.e. level, slope, and curvature. The level factor reports the same pattern as two measures of the inflation expectations, Survey of Professional Forecasters and FED Greenbook. The slope and the curvature factors are related respectively to the short-term rate and to two macroeconomic variables such as the industrial productivity and the consumption.

Our main contribution concerns the interpolation of a new term structure using the three latent factors. In an IRFs analysis, we report that more thickness of the responses means a less difference across maturities to reply a macro impact. Meanwhile, less thickness means a larger difference across maturities. This result suggests us as policy implication how a macro shock can have a different impact on the maturities of the yield curve. Moreover, we observe how all macroeconomic shocks, not only a monetary policy shocks, have implications for the whole term structure.

The remainder of this paper is organized as follows. Section 2 shows the impact of the macroeconomic variables to the term structure. Section 3 discusses the modelling of the term structure to recover latent factors. Section 4 discusses the link between the three factors (level, slope, and curvature) and the macro variables. Section 5 proposes the interpolation of the term structure, focusing on the IRFs. Section 6 closes the article. 


\section{Empirical Evidence of Macro shocks to term structure}

\subsection{Data}

The empirical analysis in this paper uses U.S. Treasury monthly data from 1984:1 to 2007:12 2 , covering the Great Moderation period. We decide to select a specific historical sample for different reasons. First, during the Great Moderation, macroeconomic variables are stable, and we can use simpler econometric tools to understand the movements and shocks impacts. Second, as our knowledge, there are not specific research studies which focus on this period.

Figure 1a presents the plot of the seven considered maturity 3, 6, 12, 24, 36, 60, and 120 months $^{3}$. Meanwhile, Figure 1b shows the complete term structure in a 3D format.

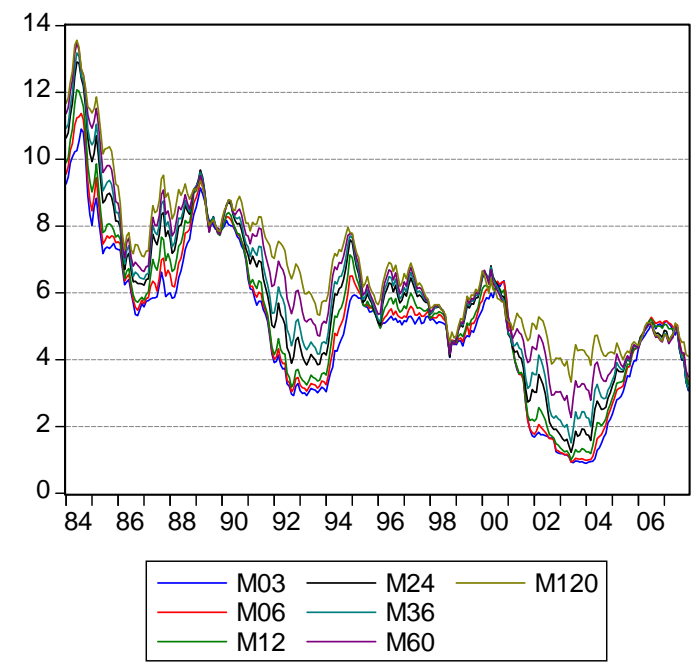

\footnotetext{
${ }^{2}$ The data are download from the database maintained by the Federal Reserve Bank of St. Louis (http://research.stlouisfed.org/fred2/).

${ }^{3}$ We select only these maturities, since they are longer and disposable for all the Great Moderation period.
} 
Figure 1a: US Treasury yields

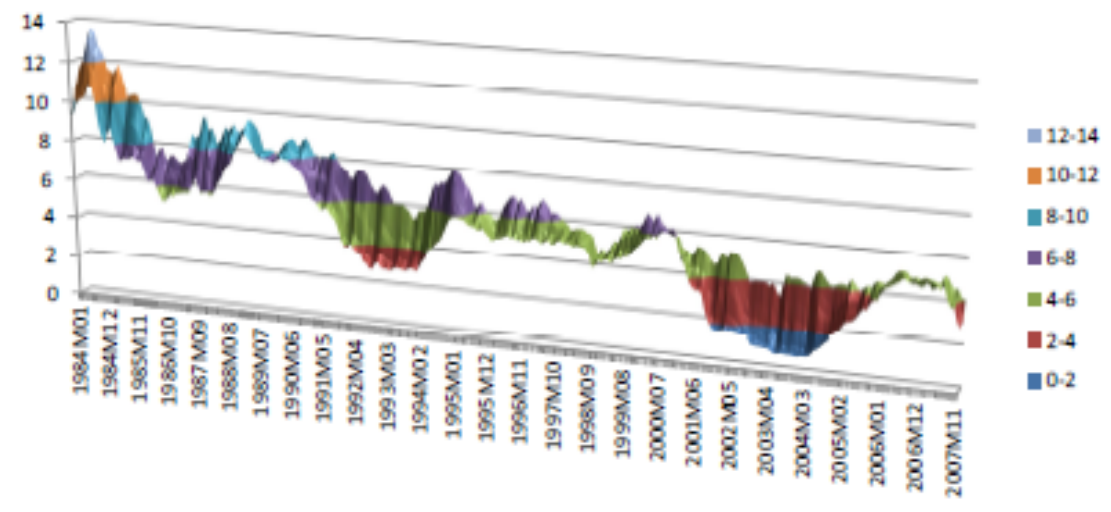

Figure 1b: US Treasury yields in 3D

Figure 1a and Figure 1b show that the U.S. yield curve exhibits sizable inter temporal variation in its level, and, although the variation in the slope and curvature is less marked, it is nonetheless evident. In particular, we can note that during the periods 1990-1995 and 2000-2005, the difference across the maturities (especially between the long term and the short term) is greater than the difference reported in other periods.

To select the key macroeconomic variables, we follow Evans and Marshall (2007). Industrial production index is detrended with both a deterministic and a stochastic trend, Inflation is measured as monthly changes in the consumer price index, the consumption is given by the personal consumption expenditures and the policy instrument is the Federal funds rate. In Figure 2, we report the macroeconomic variables already transformed and used for the empirical analysis. 

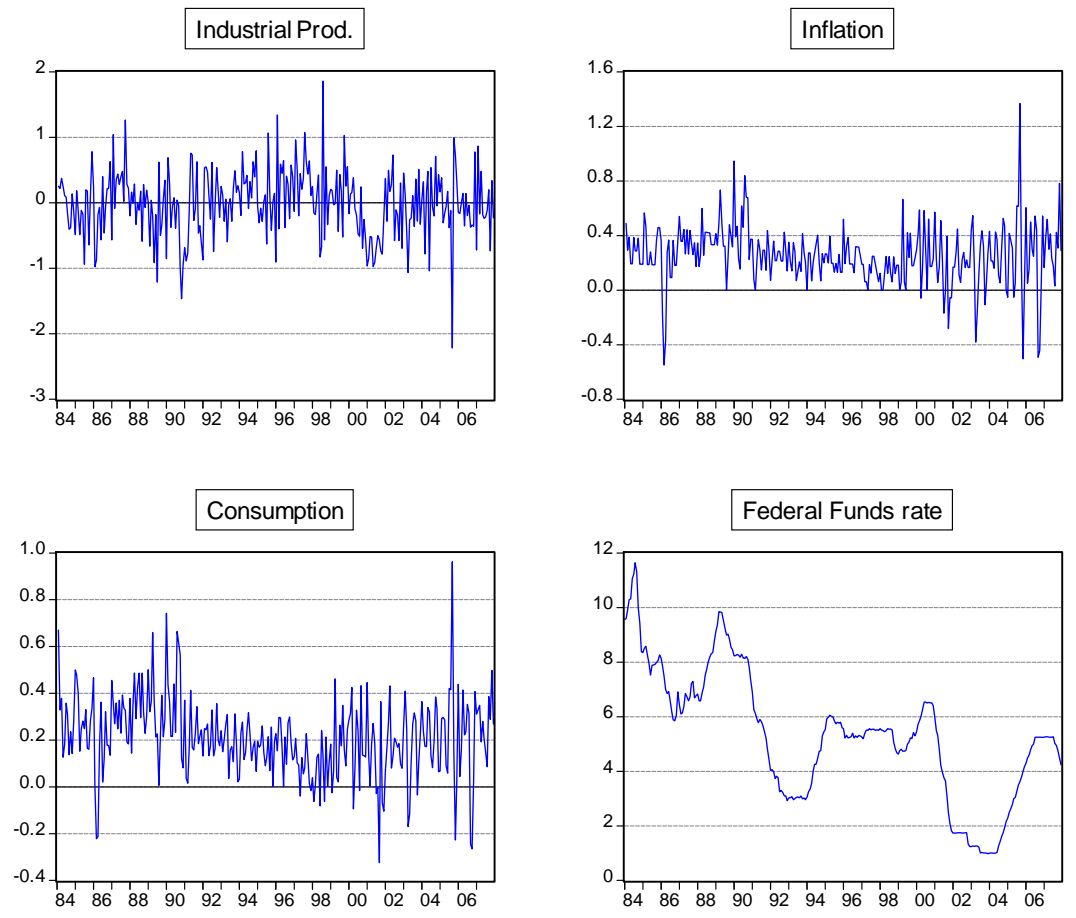

Figure 2: US Macroeconomic variables 


\subsection{Impulse-Response Functions}

To investigate about the macroeconomic determinants of the term structure, we implement a preliminary impulse-response exercise (IRFs). We estimate a VAR model with 12 lags considering the macroeconomic variables $\left(I P_{t}, I N F L_{t}, P C O M_{t}, F F R_{t}\right)$ and the seven maturities, using the Cholesky identification strategy.

Figure 3 shows the responses of each maturity to the macro variables shocks, ordered by maturity. At first glance, there is not a clear evidence across maturities. In the shorter term (m03), we note, for impulses of inflation and Federal Funds rate, an initial decreasing pattern which switches to an increasing pattern. Contrary, responses to the consumption and the industrial production index show an opposite behavior. We notice that for m24, $\mathrm{m} 36$, and $\mathrm{m} 120$ all the macroeconomic variables have an initial positive impact. 

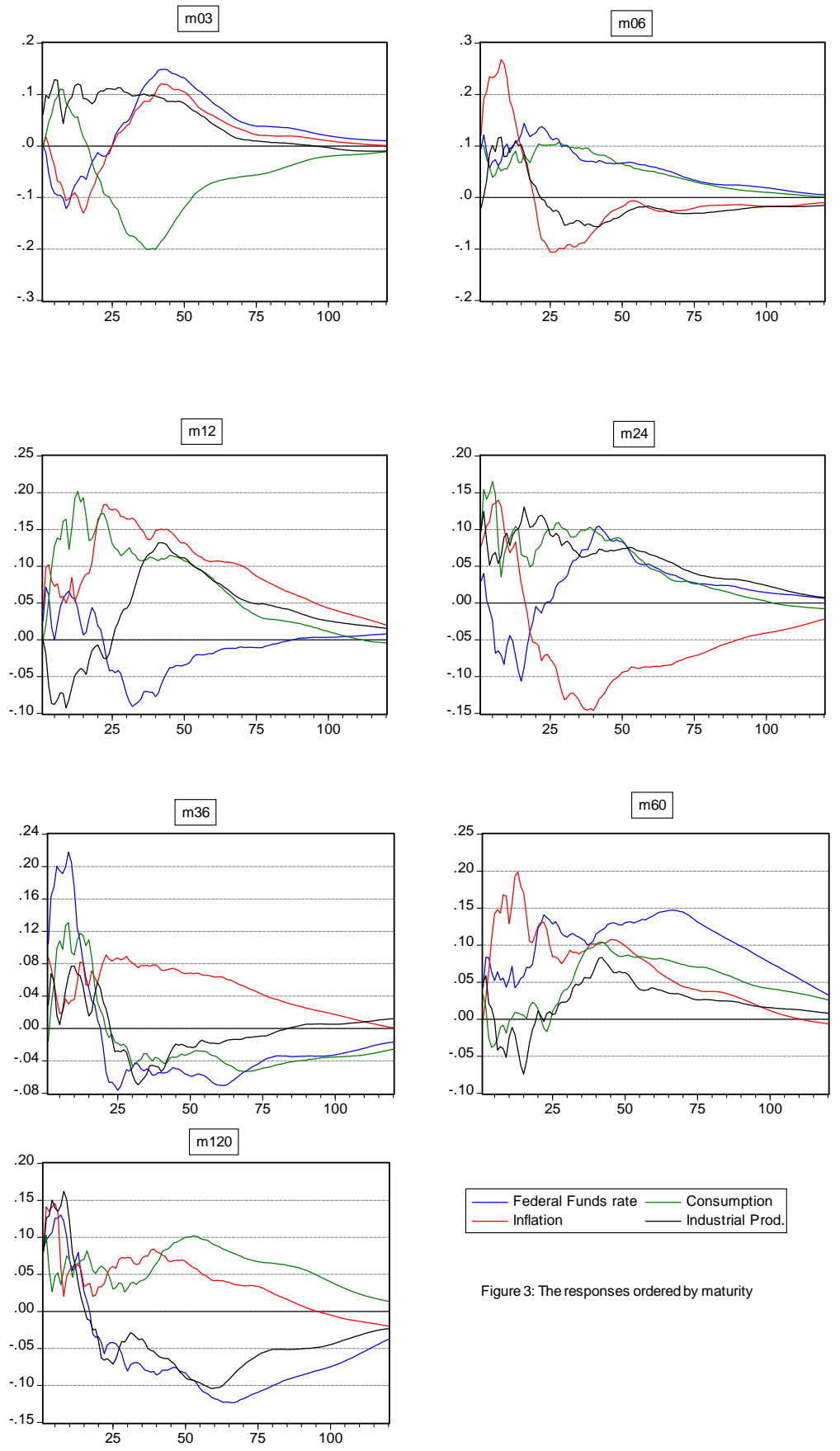

\section{- Federal Funds rate - Consumption
Inflation
Industrial Prod.}

Figure 3: The responses ordered by maturity 
Figure 4 shows the responses of each maturity to the macro variables shocks, ordered by macro variable. For the same macro variable, the response changes across different maturities and we cannot find a precise behavior. Moreover, the response to a monetary policy shock shows the most puzzling picture.
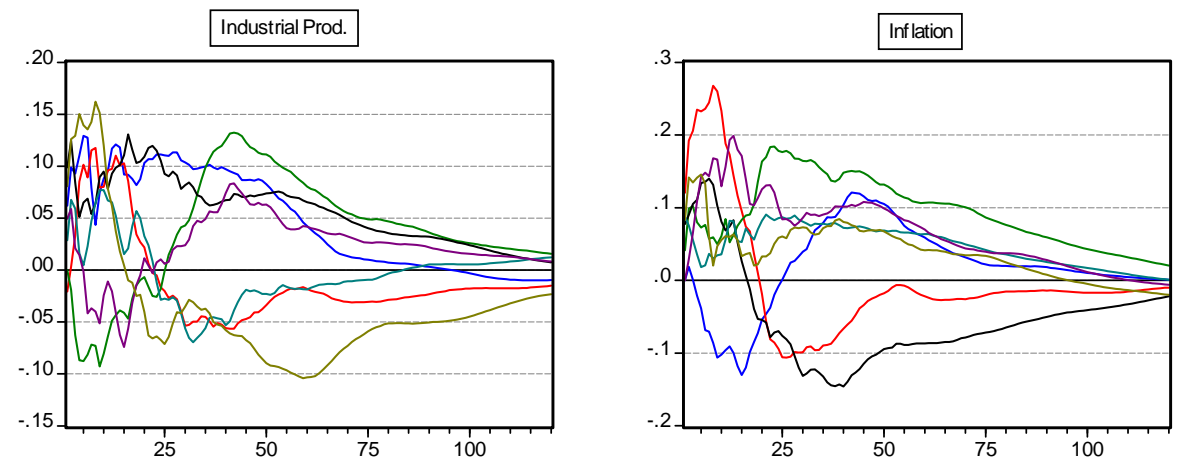

$-\mathrm{M} 03-\mathrm{M} 24-\mathrm{M} 120$
$-\mathrm{M} 66-\mathrm{M} 6$
$\mathrm{M} 12-\mathrm{M} 60$
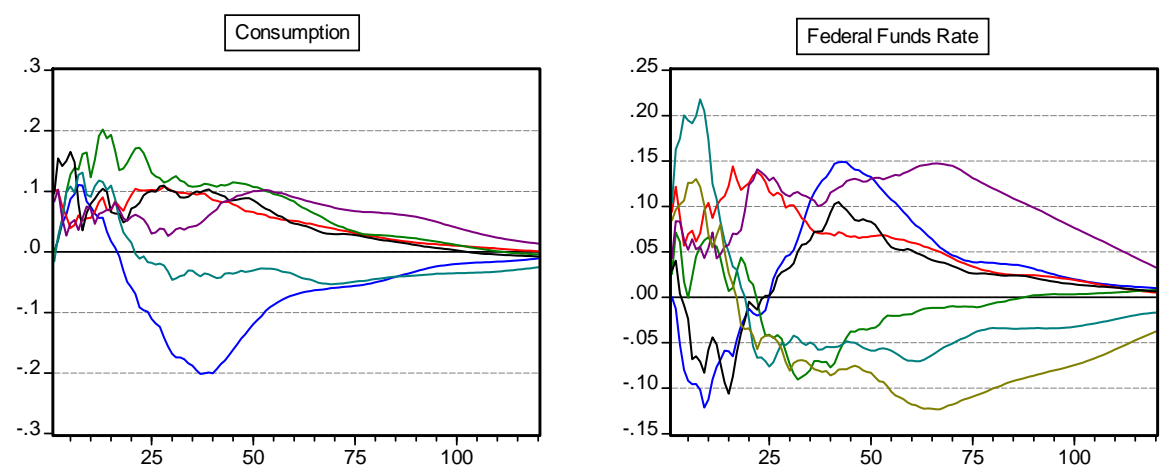

Figure 4: The responses ordered by macro variable

To understand how the overall term structure behaves, defining a common pattern among maturities, we need to improve the empirical analysis with a longer and more 
complete yield curve. Unfortunately, some maturities are not available for whole period from 1984 to 2007; for example, the yield series for 1-month Treasury bond starts only from 2001. To solve this problem, we contribute to rebuild a more complete term structure by interpolation, using the power of the latent variables.

\section{$3 \quad$ Modelling the term structure}

\subsection{The Nelson-Siegel Models}

The term structure depicts a set of yields on US Treasury securities of different maturities. The main feature of the term structure is to evidence the relationship among short-, medium-, and long-term yields. Several studies suggest a no stable relationship over time with different shapes considering different historical samples (Diebold and Li (2006), Mumtaz and Surico (2009), Gasha et al. (2010), and Medeiros and Rodriguez (2011)). The instability can be recovered using the Nelson-Siegel Models (NSMs) which reproduce the historical average sample of the term structure. As explained in Diebold and Li $(2006)^{4}$, the NSMs can account for the existence of unobservable, or latent factors and their associated factor loadings and key macroeconomic variables that underlie US Treasury security yields.

\subsubsection{Yield-Only Nelson Siegel Model}

At any given time, we have a large set of yields. As suggested by Diebold and Li (2006), we use the Nelson and Siegel (1987) functional form, which is convenient and parsimonious

\footnotetext{
${ }^{4}$ On one hand, Knez et al. (1994), Duffie and Kan (1996), and Dai and Singleton (2000) consider models in which a handful of unobserved factors explain the entire set of yields. These factors are often given labels such as "level," "slope," and "curvature," but they are not linked explicitly to macroeconomic variables. On the other hand, as explained in Ang and Piazzesi (2003) and repropose by Diebold et al. (2006), we can incorporate macro determinants into multi-factor yield curve models.
} 
three-component exponential approximation. The Nelson and Siegel (1987), as extended by Siegel and Nelson (1988), work with the forward rate curve:

$$
f_{t}(\tau)=\beta_{1 t}+\beta_{2 t} e^{-\lambda_{t} \tau}+\beta_{3 t} \lambda_{t} e^{-\lambda_{t} \tau},
$$

where $f_{t}(\tau)$ is the instantaneous forward rate, and where $\tau$ denotes maturity. The Nelson-Siegel forward rate curve can be viewed as a constant plus a Laguerre function, which is a polynomial times an exponential decay term is a popular mathematical approximating function. The corresponding yield curve, $y(\tau)$, is:

$$
y_{t}(\tau)=\beta_{1 t}+\beta_{2 t}\left(\frac{1-e^{-\lambda_{t} \tau}}{\lambda_{t} \tau}\right)+\beta_{3 t}\left(\frac{1-e^{-\lambda_{t} \tau}}{\lambda_{t} \tau}-e^{-\lambda_{t} \tau}\right)
$$

The Nelson-Siegel yield curve also corresponding to a discount curve that begins at one at zero maturity and approaches zero at infinite maturity.

The parameter $\lambda_{t}$ governs the exponential decay rate; small values of $\lambda_{t}$ mean slow decay and can better fit the curve at long maturities; instead large values of $\lambda_{t}$ mean fast decay and can better fit the curve at short. Moreover, $\lambda_{t}$ governs where the loading on $\beta_{3 t}$ achieves its maximum ${ }^{5}$.

$\beta_{1 t}, \beta_{2 t}$, and $\beta_{3 t}$ are the three latent dynamic factors called in Diebold et al. (2006) as time-varying level, slope, and curvature factors. The loading on $\beta_{1 t}$ is 1 , a constant that does not decay to zero in the limit, so the first factor can be interpreted as a longterm factor. The long-term factor $\beta_{1 t}$, for example, governs the yield curve level. As shown in Diebold and Li (2006), the level can be represented by the following combination, $\left[y_{t}(3)+y_{t}(24)+y_{t}(120)\right] / 3$. Moreover, we can note that an increase in $\beta_{1 t}$ increases all yields equally, as the loading is identical at all maturities, thereby changing the level of

\footnotetext{
${ }^{5}$ In our empirical exercise, we assume a fixed $\lambda=0.0609$ for all $t$ as used in Diebold and Li (2006).
} 
the yield curve. The loading on $\beta_{2 t}$ is $\left(\frac{1-e^{-\lambda_{t} \tau}}{\lambda_{t} \tau}\right)$, which is a function that starts at 1 but decays monotonically and quickly to 0 , so the second factor can be interpreted as a shortterm factor. The short-term factor $\beta_{2 t}$ is closely related to the yield curve slope, which we define as the three-month yield minus the ten-year yield. Moreover, we can note that an increase in $\beta_{2 t}$ increases short yields more than long yields, because the short rates load on $\beta_{2 t}$ more heavily, thereby changing the slope of the yield curve. As concerns this property, Dai and Singleton (2000) show that the three-factor models of Balduzzi et al. (1996) and Chen (1996) impose the restrictions that the instantaneous yield is an affine function of only two of the three state variables, a property shared by the Andersen and Lund (1997) three-factor nonaffine model.

The loading on $\beta_{3 t}$ is $\left(\frac{1-e^{-\lambda_{t} \tau}}{\lambda_{t} \tau}-e^{-\lambda_{t} \tau}\right)$, which starts at 0 (and is thus not short-term), increases, and then decays to zero and thus is not long-term), so the third factor can be interpreted as a medium-term factor. The medium term factor is closely related to the yield curvature which we can define as $2 y_{t}(24)-y_{t}(3)-y_{t}(120)$. Moreover, we can note that an increase in $\beta_{3 t}$ will have a little effect on very short or very long yields, which load minimally on it, but will increase medium-term yields, which load more heavily on it, thereby increasing yield curve curvature.

As argued in Diebold et al. (2006) and in Gasha et al. (2010), we use the state-space representation which provides a powerful framework for analysis and estimation of dynamic models due to the application of the Kalman filter in a maximum likelihood estimation to recover the underlying factors.

First, we re-write Eq. (2) as:

$$
y_{t}(\tau)=L_{t}+S_{t}\left(\frac{1-e^{-\lambda_{t} \tau}}{\lambda_{t} \tau}\right)+C_{t}\left(\frac{1-e^{-\lambda_{t} \tau}}{\lambda_{t} \tau}-e^{-\lambda_{t} \tau}\right),
$$

where $L_{t}, S_{t}$, and $C_{t}$ are the time-varying level, slope, curvature. If the dynamic 
movements of $L_{t}, S_{t}$, and $C_{t}$ follow a vector autoregressive process of first order, the model can be represented in a state-space format.

The transition equation, which governs the dynamics of the state vector, is:

$$
\begin{aligned}
& \left.\qquad \begin{array}{c}
L_{t}-\mu_{L} \\
S_{t}-\mu_{S} \\
C_{t}-\mu_{C}
\end{array}\right)=\left(\begin{array}{ccc}
a_{11} & a_{12} & a_{13} \\
a_{21} & a_{22} & a_{23} \\
a_{31} & a_{32} & a_{33}
\end{array}\right)\left(\begin{array}{c}
L_{t-1}-\mu_{L} \\
S_{t-1}-\mu_{S} \\
C_{t-1}-\mu_{C}
\end{array}\right)+\left(\begin{array}{c}
\eta_{t}(L) \\
\eta_{t}(S) \\
\eta_{t}(C)
\end{array}\right), \\
& t=1, \ldots, T
\end{aligned}
$$

The measurement equation, which relates a set of $N$ yields to the three unobservable factors, is:

$$
\begin{aligned}
& \left(\begin{array}{c}
y_{t}\left(\tau_{1}\right) \\
y_{t}\left(\tau_{2}\right) \\
\ldots \\
y_{t}\left(\tau_{N}\right)
\end{array}\right)=\left(\begin{array}{ccc}
1 & \frac{1-e^{-\lambda_{t} \tau_{1}}}{\lambda_{t} \tau_{1}} & \frac{1-e^{-\lambda_{t} \tau_{1}}}{\lambda_{t} \tau_{1}}-e^{-\lambda_{t} \tau_{1}} \\
1 & \frac{1-e^{-\lambda_{t} \tau_{2}}}{\lambda_{t} \tau_{2}} & \frac{1-e^{-\lambda_{t} \tau_{2}}}{\lambda_{t} \tau_{2}}-e^{-\lambda_{t} \tau_{2}} \\
1 & \ldots & \ldots \\
1 & \frac{1-e^{-\lambda_{t} \tau_{N}}}{\lambda_{t} \tau_{N}} & \frac{1-e^{-\lambda_{t} \tau_{N}}}{\lambda_{t} \tau_{N}}-e^{-\lambda_{t} \tau_{N}}
\end{array}\right)\left(\begin{array}{c}
L_{t} \\
S_{t} \\
C_{t}
\end{array}\right)+\left(\begin{array}{c}
\varepsilon_{t}\left(\tau_{1}\right) \\
\varepsilon_{t}\left(\tau_{2}\right) \\
\ldots \\
\varepsilon_{t}\left(\tau_{N}\right)
\end{array}\right), \\
& t=1, \ldots, T .
\end{aligned}
$$

The state-space representation in a generic format is as follows:

$$
\begin{aligned}
\left(f_{t}-\mu\right) & =A\left(f_{t-1}-\mu\right)+\eta_{t} \\
y_{t} & =\Lambda f_{t}+\varepsilon_{t} .
\end{aligned}
$$

where in the transition equation, Eq. (6), the unobservable vector $f_{t}=\left(L_{t}, S_{t}, C_{t}\right)$, the mean state vector $\mu$ is a $3 \times 1$ vector of coefficients, the transition matrix $A$ is a $3 \times 3$ matrix of coefficients, $\eta_{t}$ is a white noise transition disturbance with a $3 \times 3$ non-diagonal 
covariance matrix $Q$. Instead, in the measurement equation, Eq. (7), vector of yields $y_{t}$ contains $N$ maturities, the measurement matrix $\Lambda$ is an $N \times 3$ matrix whose columns are the loadings associated with the respective factors, and $\varepsilon_{t}$ is a white noise measurement disturbance with an $N \times N$ diagonal covariance matrix $H$.

As shown in Diebold et al. (2006), for non linear least-squares optimality of the Kalman filter, we require the white noise transition and measurement disturbances be orthogonal to each other and to the initial state:

$$
\begin{aligned}
& \left(\begin{array}{c}
\eta_{t} \\
\varepsilon_{t}
\end{array}\right) \sim W N\left[\left(\begin{array}{l}
0 \\
0
\end{array}\right),\left(\begin{array}{ll}
Q & 0 \\
0 & H
\end{array}\right)\right], \\
& E\left(f_{0} \eta_{t}^{\prime}\right)=0 \text { and } E\left(f_{0} \varepsilon_{t}^{\prime}\right)=0
\end{aligned}
$$

Diebold et al. (2006) assume that the $H$ matrix is diagonal and the $Q$ matrix is nondiagonal. The assumption of a diagonal $H$ matrix, which implies that the deviations of yields of different maturities from the yield curve are uncorrelated, is quite standard ${ }^{6}$. The assumption of an unrestricted $Q$ matrix allows the shocks to the three term structure factors to be correlated

\subsubsection{Yield-Macro Nelson Siegel Model}

In the Yield-Macro Nelson Siegel model, we emphasize on the relationships among $\widehat{L}_{t}, \widehat{S}_{t}$, and $\widehat{C}_{t}$ and the macroeconomic variables. As follows the approach proposed by Diebold et al. (2006) to use a state-space representation to incorporate macroeconomic factors in a latent factor model of the term structure to analyze the potential bidirectional feedback

\footnotetext{
${ }^{6}$ For example, to estimate the no-arbitrage term structure models, "measurement error" is added to the observed yields. The same assumption is required for computational tractability given the large number of observed yields used.
} 
between the term structure and the macroeconomic variables. We include in the state vector the four key variables: industrial production index $\left(I P_{t}\right)$, annual price inflation $\left(I N F L_{t}\right)$, personal consumption $\left(P C O M_{t}\right)$, and the Federal Funds rate $\left(F F R_{t}\right)$.

The extension of the yields-only model adds the three macroeconomic variables to the set of the state variables and replace Eqs. (6) - (8) with:

$$
\begin{aligned}
\left(f_{t}^{\prime}-\mu\right) & =A\left(f_{t-1}^{\prime}-\mu\right)+\eta_{t} \\
y_{t} & =\Lambda f_{t}^{\prime}+\varepsilon_{t} \\
\left(\begin{array}{c}
\eta_{t} \\
\varepsilon_{t}
\end{array}\right) & \sim W N\left[\left(\begin{array}{l}
0 \\
0
\end{array}\right),\left(\begin{array}{ll}
Q & 0 \\
0 & H
\end{array}\right)\right] .
\end{aligned}
$$

where $f_{t}^{\prime}=\left(L_{t}, S_{t}, C_{t}, I P_{t}, I N F L_{t}, P C O M_{t}, F F R_{t}\right)$ and the dimension of $A, \mu, \eta_{t}$ and $Q$ are increased accordingly to $7 \times 1,7 \times 7$, and $7 \times 1$, respectively.

\section{Extracted Latent Factors}

Using the NSMs, we extract three latent factors using the state-space representation augmented by the macro variables as described in Eq. (10) and Eq. (11). For more technical details, see Diebold et al. (2006) and Gasha et al. (2010). The three factors, level, slope, and curvature, are extracted considering the US Treasury yield curve with maturities of 3 , $6,12,24,36,60$, and 120 months.

Figure 5 shows the comparison between the factors extracted using the NSM and their "empirical counterparts". The empirical counterparts of the factors can be thought of as crude proxies for the level, slope and curvature of the yield curve and following Diebold and $\mathrm{Li}(2006)$ are calculated as: 
Level: $\left[y_{t}(3)+y_{t}(24)+y_{t}(120)\right] / 3$

Slope: $y_{t}(3)-y_{t}(120)$

Curvature: $2 y_{t}(24)-y_{t}(3)-y_{t}(120)$.

In the top left panel of Figure 5, we show the level factor (blue line) and the counterpart (red line). The correlation between the level factor and its counterpart is around 0.90, which is similar to the numbers reported by Diebold et al. (2006) and Mumtaz and Surico (2009) 7. The bottom left panel reports two measure of inflation expectations: the Survey of Professional Forecasters (SPF) and the FED Greenbook 1 year head as shown in Mumtaz and Surico $(2009)^{8}$. Comparing the level factors and the two measures of expectations, we can recognize a strong link between the level of the yield curve and inflation expectations (see Kozicki and Tinsley (2001); Hordahl et al. (2006)).

In the middle of the panel, we report the slope factor which is correlated with the empirical counterpart with a number around 0.99. In the Nelson-Siegel model, the slope is identified as the factor that is loaded more heavily by yields of short maturities, hence we can find a similar pattern between slope and Federal Funds rate. Lastly, the correlation between the curvature factor and the empirical counterpart is around 0.98 and we can recognize a similar behaviour between the curvature and the industrial productivity index.

\footnotetext{
${ }^{7}$ Mumtaz and Surico (2009) report a correlation around 0.90 with time-varying coefficients and around 0.80 without time-varying coefficients for the sample period from 1970 to 2000. Diebold et al. (2006) consider a range from 1985 to 2000 with a correlation around 0.97 .

${ }^{8}$ These forecasts for the two measures of expectations are available at quarterly frequency on the web site of the Federal Reserve Bank of Philadelphia, respectively at http://www.phil.frb.org/econ/spf/spfmed.html (SPF), and http://www.phil.frb.org/econ/forecast/croushoresdatasets.html
}

(Greenbook). 

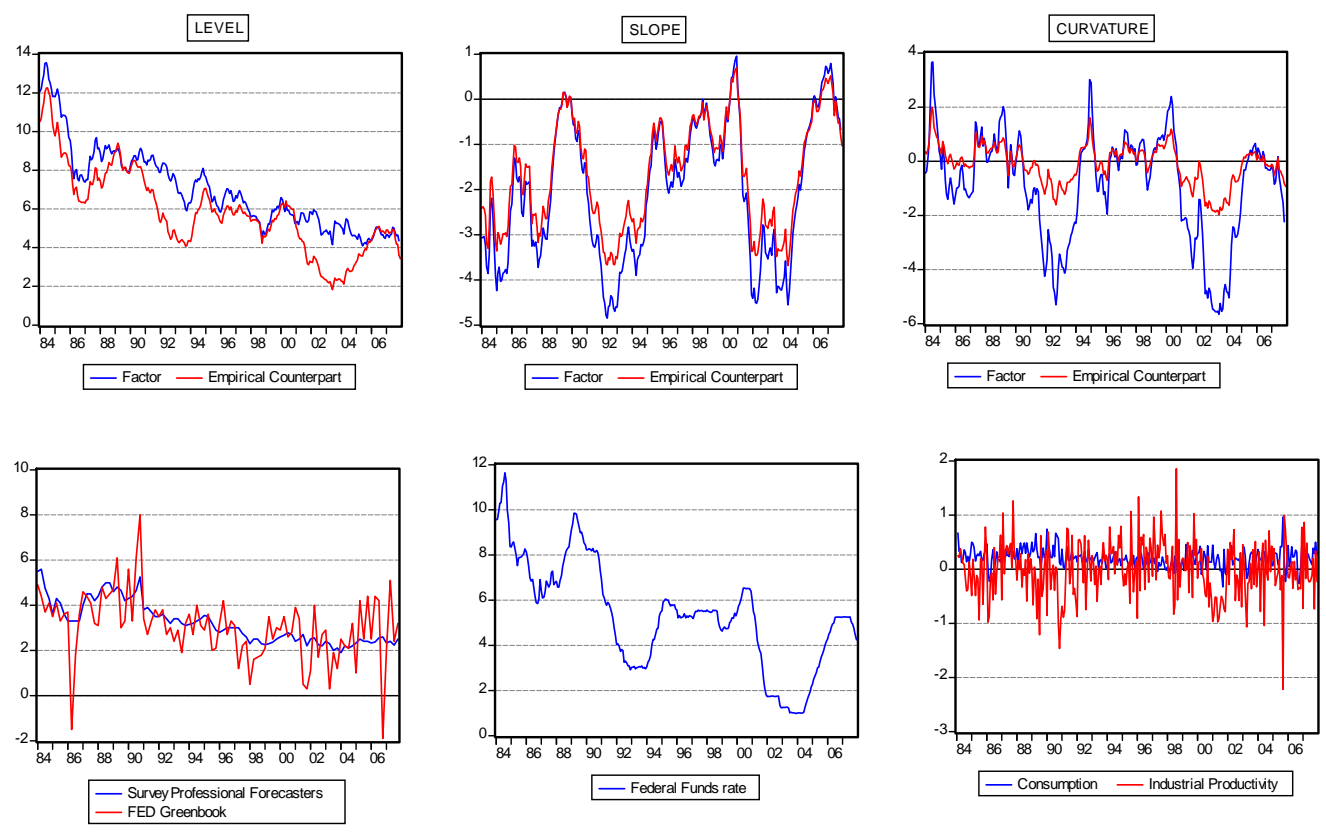

Figure 5. Factors and empirical counterpart

\section{$5 \quad$ Interpolating the term structure}

The three extracted factors are used to interpolate a complete term structure using the Eq $(2)$ :

$$
y_{t}(\tau)=\beta_{1 t}+\beta_{2 t}\left(\frac{1-e^{-\lambda_{t} \tau}}{\lambda_{t} \tau}\right)+\beta_{3 t}\left(\frac{1-e^{-\lambda_{t} \tau}}{\lambda_{t} \tau}-e^{-\lambda_{t} \tau}\right)
$$

where $\beta_{1 t}, \beta_{2 t}$, and $\beta_{3 t}$ are substituted by the three factors estimated and $\tau$ is substi- 
tuted by the maturity for each corresponding yield ${ }^{9}$.

Figure 6 compares the observed maturities with the maturities interpolated with the latent factors. We note that the reproduction of the yields is very close to the observed yields and the correlation is over 0.95 for each maturity.
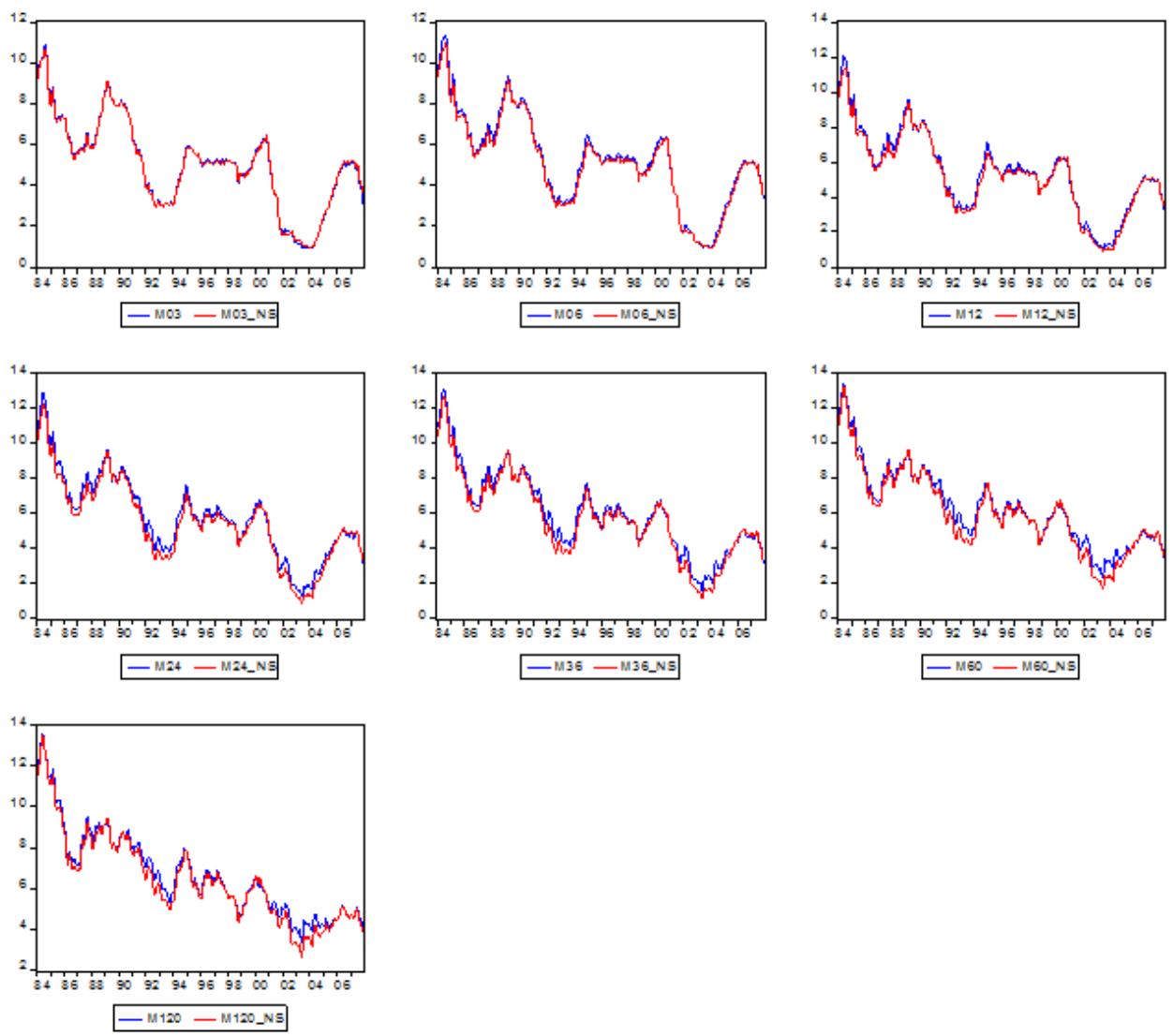

Figure 6: Comparison observed and interpolated yields

Figure $7 \mathrm{a}$ and Figure 7b show the new term structure with 21 maturities and we can

\footnotetext{
${ }^{9}$ We repeat the interpolation for 21 maturities $(1,2,3,4,5,6,7,8,9,10,11,12,24,36,48,60,72,96$, 108, 120 months).
} 
note a more compact behaviour of the yields in both plots.

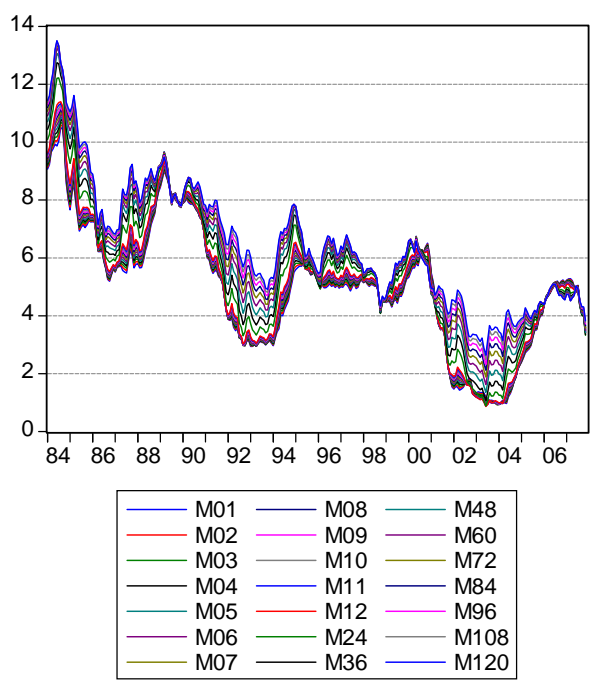

Figure 7a: New term structure

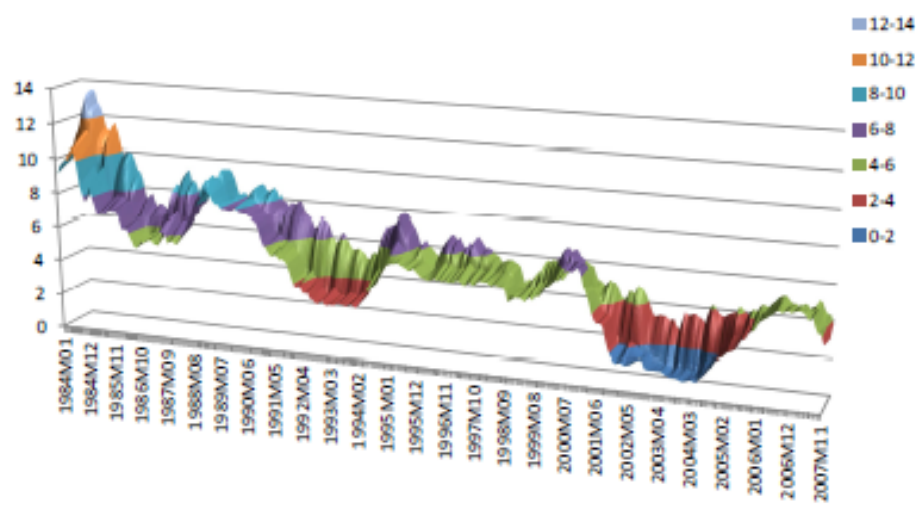

Figure 7b: New term structure in 3D 


\subsection{Impulse-Response Functions}

We repeat the preliminary analysis of the responses of each maturity to the macro shocks, using the term structure with 21 yields.

Figure 8 is the corresponding picture presented by Figure 4, but with 21 maturities instead of 7. All the plots show how in the short period the responses are compact over maturities. However, between 20 and 60 months, the responses become less thickness, reporting a wide difference from a short yield to a medium yield. The only exception is given by a monetary policy shock which reports a wide difference in responses in the short and in the medium period up 60 months. Another interesting aspect is that for all shocks, except for the consumption shock, around 25-30 months, there is point in which all the responses are equal. In the medium period, we note that the consumption shows the smaller distance between the short and the long yields. Instead, the inflation is the variable which has a huge impact across different maturities showing two episodes of wider responses of the maturities. The industrial productivity index as the Federal Funds rate evidence only one big episode of a wider response between the short and the long yields, reporting a similar behavior. In the long period, after 60 months, we notice a compact pattern for all responses. Consequently, we can affirm how the macroeconomic shocks have implications for the whole term structure, this also means that the entire yield curve, not

just the short rate, contains potentially valuable information about not only the monetary policy shifts, but also about an industrial productivity shock, inflation and consumptions shocks. 

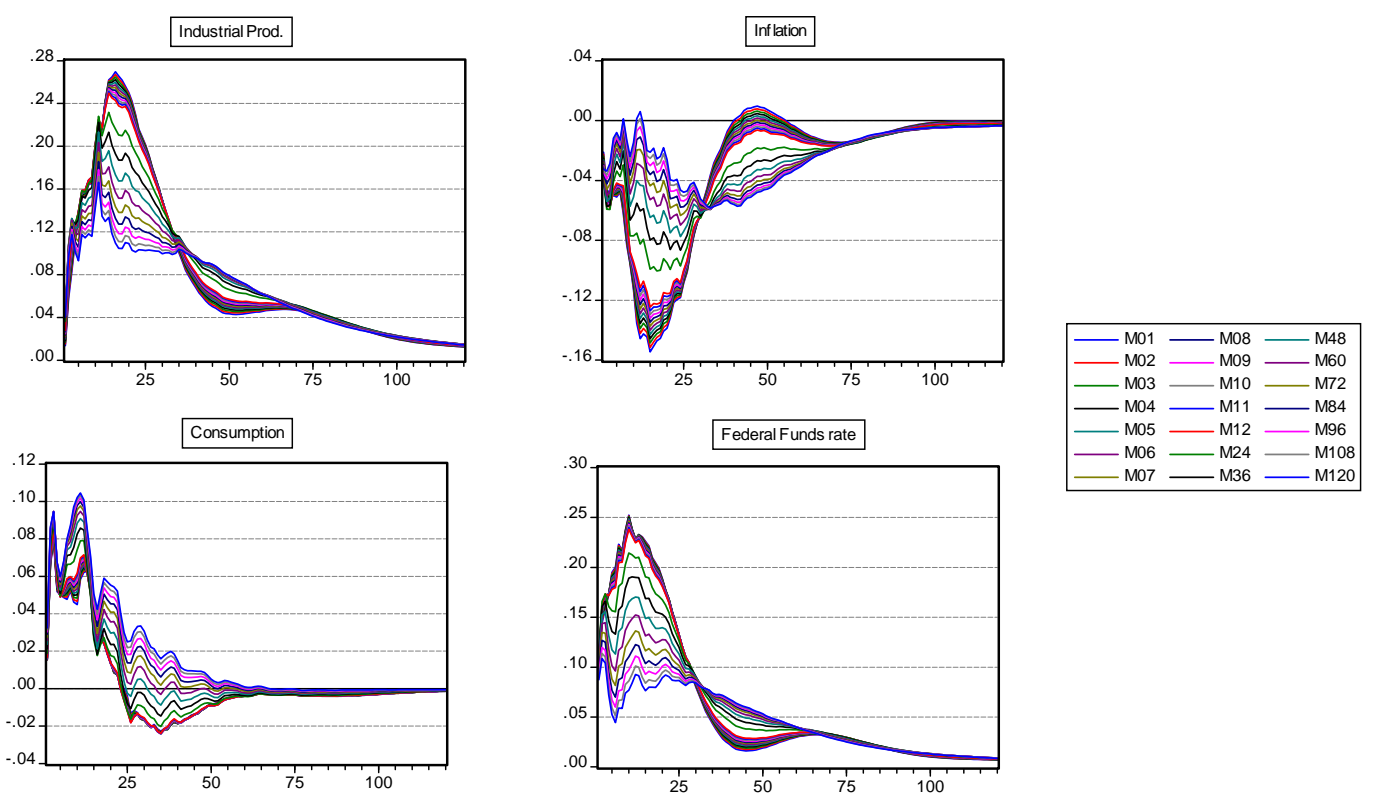

Figure 8: IRF from the interpolated term structure

\section{Concluding Remarks}

We study the impact of the macroeconomic determinants on the US term structure during the Great Moderation period. To complete the yield curve, we extract, using a NelsonSiegel Model, three latent factors, level, slope, and curvature. The three factors, even if they come from the yield curve, can be associated to relevant macroeconomic variables, such as, inflation, industrial productivity index, Federal Funds rate, and inflation. Our contribution is to interpolate the term structure with the latent factors to investigate the impact of each macroeconomic variable on each maturity of the yield curve.

As policy implication, the interpolated term structure suggests us how all the macro 
shocks, not only the monetary policy shifts, impact on the overall yield curve. Moreover, the responses are less thickness reporting a wide difference in response from a short period to a medium period, between 20 and 60 months.

\section{References}

[1] Andersen TG, Lund J. 1997. Stochastic volatility and mean drift in the short term interest rate diffusion: source of steepness, level and curvature in the yield curve. Working Paper 214, Department of Finance, Kellogg School, Northwestern University.

[2] Ang A, Piazzesi M. 2003. A no-arbitrage vector autoregression of term structure dynamics with macroeconomic and latent variables. Journal of Monetary Economics, Elsevier, vol. 50(4), pages 745-787.

[3] Balduzzi P, Das SR, Foresi S, Sundaram R. 1996. A simple approach to three-factor affine term structure models. Journal of Fixed Income 6, 43-53.

[4] Baz J, Chacko G. 2004. Financial Derivatives: Pricing, Applications and Mathematics (Cambridge, United Kingdom: Cambridge University Press).

[5] Bianchi F, Mumtaz H, Surico P. 2009. The great moderation of the term structure of UK interest rates. Journal of Monetary Economics 56: 856-871.

[6] Bolder DJ. 2001. Affine term structure Models: Theory and Implementation. Working Paper 2001-15 (Ottawa: Bank of Canada).

[7] Cenesizoglu T, Larocque D, Normandin M. 2013. Conventional Monetary Policy and the Term Structure of Interest Rates during the Financial Crisis. mimeo.

[8] Chen L. 1996. Stochastic Mean and Stochastic Volatility-A Three Factor Model of 
the Term Structure of Interest Rates and its Application to the Pricing of Interest Rate Derivatives. Blackwell Publishers, London.

[9] Contessi S, De Pace P, Guidolin M. 2014. How Did the Financial Crisis Alter the Correlations of U.S. Yield Spreads?, Journal of Empirical Finance forthcoming.

[10] Dai, Q, Singleton, K. 2000. Specification analysis of affine term structure models. Journal of Finance 55, 1943-1978.

[11] De Pace P, Weber K. 2013. High yield spreads, real economic activity, and the financial accelerator. Economics Letters, Volume 121, Issue 3, December 2013, Pages 346355.

[12] Diebold FX, Li C. 2006. Forecasting the term structure of government bond yields. Journal of Econometrics 130: 337-364.

[13] Diebold, FX, Piazzesi M, Rudebusch, GD. 2005. Modeling Bond Yields in Finance and Macroeconomics. American Economic Review Papers and Proceedings, 95, $415-420$.

[14] Diebold FX, Rudebusch GD, Aruoba SB. 2006. The macroeconomy and the yield curve: a dynamic latent factor approach. Journal of Econometrics 131: 309-338.

[15] Duffie D, Kan R. 1996. A yield-factor model of interest rates. Mathematical Finance $6,379-406$.

[16] Evans CL, Marshall D. 2007. Economic determinants of the nominal treasury yield curve. Journal of Monetary Economics 54: 1986-2003.

[17] Fama EF, Bliss RR. 1987. The information in long-maturity forward rates. American Economic Review 77(4): 680-692.

[18] Gasha G, He Y, Medeiros C, Rodriguez M, Salvati J, Yi J. 2010. On the Estimation of Term Structure Models and An Application to the United States. IMF Working 
Paper. WP/10/258.

[19] Hordahl P, Tristani O, Vestin D. 2006. A joint econometric model of macroeconomic and term structure dynamics. Journal of Econometrics 127(1-2): 405-444.

[20] Knez P, Litterman R, Scheinkman J. 1994. Exploration into factors explaining money market returns. Journal of Finance 49, 1861-1882.

[21] Kozicki, Tinsley PA. 2001. Shifting endpoints in the term structure of interest rates. Journal of Monetary Economics 47(3): 613-652.

[22] Mumtaz H, Surico P. 2009. Time-varying yield curve dynamics and monetary policy. Journal of Applied Econometrics 24: 895-913.

[23] Medeiros C, Rodríguez M. 2011. The Dynamics of the Term Structure of Interest Rates in the United States in Light of the Financial Crisis of 2007-10. IMF Working Paper. WP $/ 11 / 84$.

[24] Nelson CR, Siegel AF. 1987. Parsimonious modeling of yield curves. Journal of Business 60(4): 473-489.

[25] Reinhart C, Rogoff K. 2009. The Aftermath of Financial Crises. The American Economic Review Vol. 99, No. 2, Papers and Proceedings of the One Hundred TwentyFirst Meeting of the American Economic Association (May, 2009) , pp. 466-472.

[26] Siegel AF, Nelson CR. 1988. Long-term behavior of yield curves. Journal of Financial and Quantitative Analysis 23, 105-110.

[27] Van Deventer DR, Imai K, Mesler M. 2005. Advanced Financial Risk Management (Singapore: John Wiley \& Sons (Asia) Pte Ltd). 